\title{
Interpreting filtration-based suffusion criteria using micro-Computed Tomography and laboratory filter tests
}

\author{
H.F. Taylor \\ Imperial College London \\ C. O’Sullivan \\ Imperial College London \\ W. W. Sim \\ Atkins
}

ABSTRACT: In a cohesionless soil containing a mixture of coarse and fine particles, suffusion is the erosion of the fine particles which can be transported through the void space between the coarse particles. Two commonly used criteria for suffusion are Kézdi (1979) and Kenney \& Lau (1985), both of which were determined empirically and both include a limiting factor of 4 between fine and coarse particle sizes. This factor of 4 is often interpreted based on simple filter criteria, as the ratio between the size of coarse particles and the size of constrictions in the void space, with some factor of safety. To fully understand the suffusion mechanism it is necessary to consider the size of void constrictions, but also how far fine particles can move before they are retained and the potential for the fine material to self-filter. Several researchers have used empirical and numerical results to show that the criteria are conservative, however these studies could not clearly distinguish between the issues of constriction size, particle movement and self-filtration. This paper uses micro-Computed Tomography (microCT) images to examine the underlying filter criteria, by measuring constriction sizes relative to particle sizes for three materials. Laboratory filter tests, with minimal selffiltration, are then presented to observe particle movement. The results provide a new means to interpret the physical significance of the filter criteria, on which suffusion criteria are based.

\section{INTRODUCTION}

\subsection{Background}

Roughly half of all embankment dam failures occur as a result of internal erosion (Foster et al. 2000), which can take the form of concentrated leaks, contact erosion, backward erosion or suffusion. Suffusion, sometimes referred to as internal instability, occurs in cohesionless materials with a mixture of coarse and fine particles, where the fine particles can be freely transported through the void space between the coarse particles. This requires the fine particles to be loose, i.e. under low effective stresses, which only occurs when fine particles make up approximately $<35 \%$ of the total mass (Skempton \& Brogan 1994; Shire et al. 2014). Loose fines must also be able to fit through the narrowest points in the void space, referred to as constrictions. This issue of whether fine particles can fit between coarse particles is also considered when specifying granular filters, where a layer of coarse granular material (the filter) must retain a separate layer of fine particulate material (the base) and for which the classical design criterion is:

$D_{15} \leq 4 d_{85}$ where $D_{15}$ is the particle diameter with $15 \%$ passing in the filter and $d_{85}$ is the particle diameter with $85 \%$ passing in the base. This criterion was proposed by Terzaghi based on laboratory filter tests and he justified the results on the basis that "the pore size of a broadly-graded filter comprises at maximum $1 / 5$ th of the diameter of the biggest grain of the finest fraction of the filter materials" (Fannin 2008). The smaller constrictions in the filter will govern its performance and if the larger particles in the base are retained they will start to act as filters for the smaller base particles (referred to as self-filtration). Hence Terzaghi's statement suggests there should be a limiting factor of 5 between the larger base material $\left(d_{85}\right)$ and the smaller filter material $\left(D_{15}\right)$ and hence the value of 4, rather than 5, in Equation 1 implies a factor of safety.

Shire et al. (2012) used discrete element modelling (DEM) to measure the size of constrictions between spherical particles. Their results showed that $D_{15} / 4$ corresponds to roughly the $50^{\text {th }}$ percentile of the constriction size distribution (CSD) of a filter and hence $D_{15} / 4$ gives a conservative estimate of the smaller constriction sizes in the filter. 


\subsection{Kézdi criterion}

Figure 1(a) shows a schematic particle size distribution (PSD) for a gap graded material, containing fine material and coarse material with a gap across the intermediate sizes. Kézdi (1979) performed laboratory tests on gap graded materials and found that suffusion did not occur provided that Terzaghi's filter criterion (Equation 1) was satisfied between the coarse and fine fractions of the material.

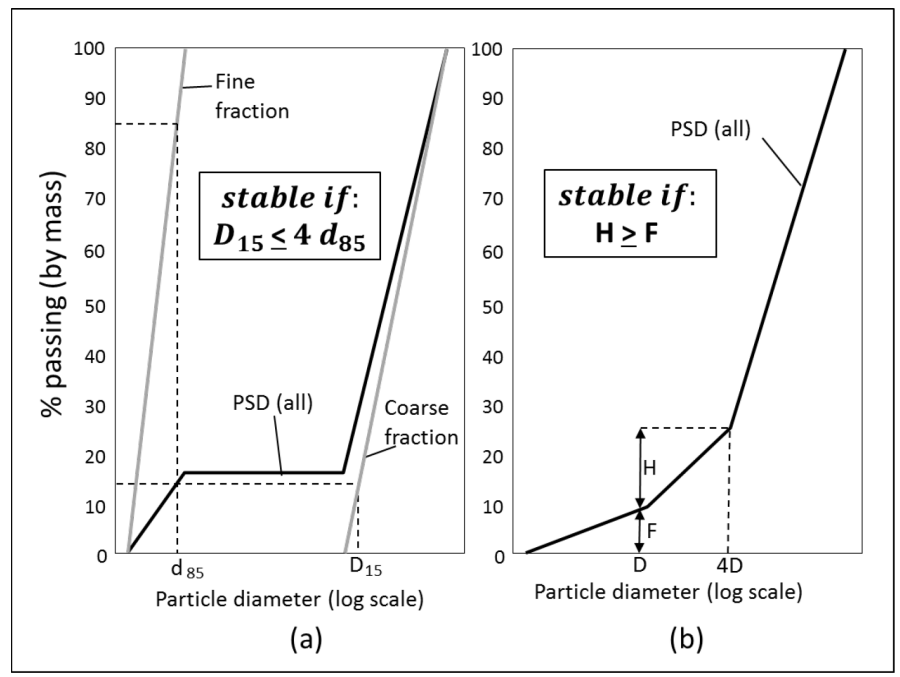

Figure 1: Schematic PSDs showing a) Gap graded material and Kézdi (1979) criterion, b) Broadly graded material and Kenney \& Lau (1985) criterion

Fannin \& Moffat (2006) performed laboratory tests on a range of gap and broadly graded PSDs and reported that suffusion did not occur for $\mathrm{D}_{15} / \mathrm{d}_{85}$ ratios of $\leq 5$, but even at higher ratios suffusion only occurred at extremely high applied hydraulic gradients, again suggesting the criterion is conservative. Tests on filters by Bertram (1940) suggested a limiting $\mathrm{D}_{15} / \mathrm{d}_{85}$ value of 6 .

Kézdi also used simple analytical models to estimate the size of individual void constrictions between spherical particles and supported the notion that Terzaghi's use of the factor 4 includes a factor of safety.

\subsection{Kenney \& Lau criterion}

One issue with the Kézdi criterion is that the PSD must be split into fine and coarse fractions. In the case of a broadly graded material (Figure 1(b)) the distinction between fine and coarse is subjective. Kenney \& Lau (1985) performed laboratory tests on a range of broadly graded materials and proposed the method as shown in Figure 1(b), in which any size, $D$, can be assessed to ensure there is sufficient material between sizes $D$ and $4 D$ to retain it. This check is performed for every size up to $20 \%$ passing. Again the decision to assess material up to 4 times $D$ is justified based on the ratio of particle sizes to the void constrictions they form. It should be noted that in the materials which Kenney \& Lau (1985) defined as unstable, suffusion only occurred when extremely high hydraulic gradients were applied and the apparatus was agitated with a hammer.

Ronnqvist (2015) analysed laboratory data and case studies on a range of glacial tills and found that the Kenney \& Lau criterion correctly identified almost $80 \%$ of cases where suffusion occurred. However suffusion only occurred in roughly half of all the cases where the criterion suggested the potential for suffusion.

\subsection{Outline of this study}

At first glance both the Kézdi (1979) and Kenney \& Lau (1985) criteria appear to have a clear physical significance, being based on filter rules with the factor 4 representing a ratio of the size of coarse particles to the size of fines which can fit between them, with some factor of safety. However the physical mechanisms of filtration or suffusion in the laboratory does not just depend on a single pairing of particle size to constriction size, but involve a large range of fine particles moving through a large range of constrictions. To fully understand the physical significance of the factor 4 in the underlying filter criteria (and hence how conservative they are), three distinct issues need to be considered:

- The actual ratio of particle sizes to constriction sizes

- The movement of fine particles before they encounter a suitably small constriction.

- The importance of self-filtration by the fine particles.

This paper presents data from samples of sands and glass beads with which it is possible to isolate these three issues for relatively uniform materials. These materials are intended to represent either a granular filter, or the coarse fraction of a gap graded material. High resolution micro-Computed Tomography (microCT) images are used to provide accurate measurements of particle and constriction sizes, for direct comparison. Laboratory filter tests, designed to minimize self-filtration, are used to demonstrate how, even in an effective filter, there is significant movement of fine particles before they are retained, but also that self-filtration of fines is crucial.

\section{VOID CONSTRICITON SIZES}

\subsection{Micro-Computed Tomography imaging}

Three materials were considered in this study; two samples of sub-angular Leighton Buzzard sand with coefficient of uniformity, $C_{u}$, values of 3 and 1.5, and a third sample was made from borosilicate glass beads with a $C_{u}$ value of 3 . The samples are referred to as ' $\mathrm{Cu} 3$ ', ' $\mathrm{Cu} 1.5$ ' and 'GBCu3' respectively.

MicroCT imaging requires small samples to achieve sufficient resolution for individual particles 
to be measured in detail (Cnudde \& Boone 2013). These small samples were produced using the methodology described in Fonseca et al. (2012). 38mm diameter samples were prepared by dry deposition (Ishihara 1993) in the triaxial apparatus; a cell air pressure of $30 \mathrm{kPa}$ was applied; the samples were impregnated with epoxy resin and, after 24 hours curing time, $9 \mathrm{~mm}$ diameter sub-samples were cored from the centre of each triaxial sample.

The $9 \mathrm{~mm}$ cores were scanned using a Nikon XTH-225 microCT scanner at Queen Mary University London, producing the images shown in Figure 2, with voxels (volume pixels) measuring approximately $10 \mu \mathrm{m} \times 10 \mu \mathrm{m} \times 10 \mu \mathrm{m}$. The greyscale in Figure 2 indicates the level of x-ray attenuation, which is directly proportional to density, hence lighter shades represent denser material.

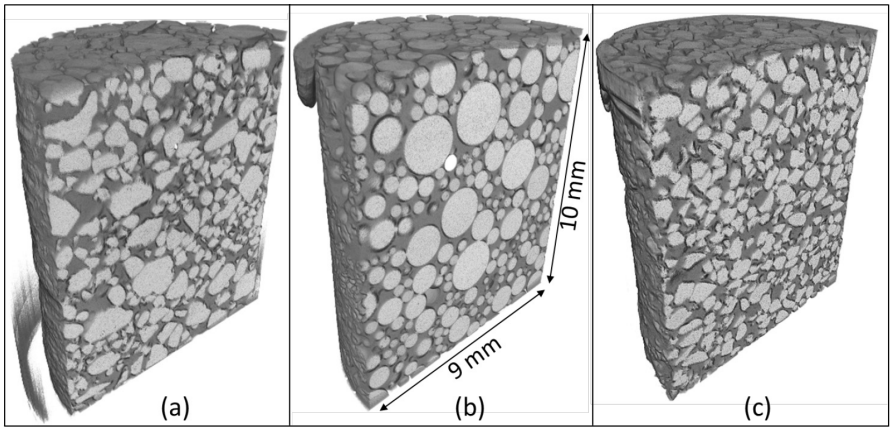

Figure 2: MicroCT images a) $\mathrm{Cu} 3$, b) $\mathrm{GBCu} 3$, c) $\mathrm{Cu} 1.5$

The microCT images were processed using median filtering and the Otsu (1979) thresholding method to produce binary images where each voxel is defined as either solid or void. Watershed segmentation was applied first to the solid phase to identify each individual particle (as described in Fonseca et al. (2012)), so that each particle could be measured and the PSD determined. Watershed segmentation was also applied to the void phase (as described in Taylor et al. (2015)) to identify individual void regions, as shown in Figure 3, where each void region is assigned a different grey shade. It is important to note that this is a 2D 'slice' from a 3D image and interpreting voids or constrictions from $2 \mathrm{D}$ images can be misleading. All analyses discussed here were all based on full 3D geometry.

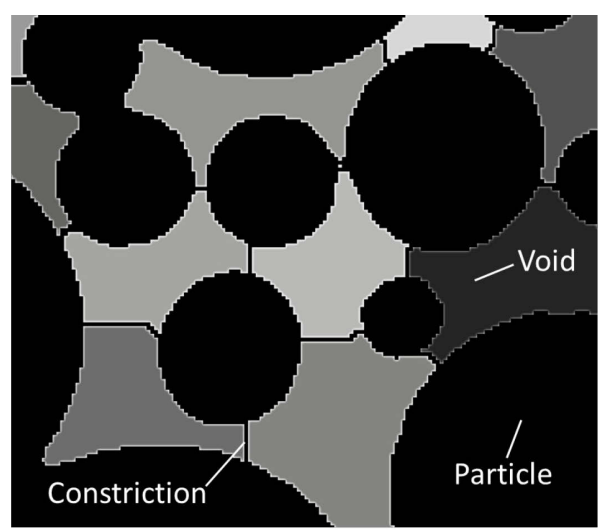

Figure 3: Blow-up of 2D slice from GBCu3 image, showing individual void regions and constrictions
Vincens et al. (2014) outlined a number of methods to measure constriction sizes from micro-CT images, DEM simulations or laboratory tests. In this study the constrictions (the narrowest points in the void space) were identified as the boundaries between individual void regions, indicated by the black lines in Figure 3, and the size of each constriction was measured using the method described by Taylor et al. (2015), where the constriction size refers to the diameter of the largest sphere which can pass the constriction.

\subsection{Results: CSDs vs PSDs}

Figure 4 presents CSDs (solid grey lines) and PSDs (solid black lines) for the three materials: note that percentage passing is presented 'by mass' for PSDs and 'by number' for CSDs, as constrictions have no volume. To examine the physical significance of the Kézdi (1979) and Kenney \& Lau (1985) criteria, dashed lines are shown in Figure 4 representing particle diameters divided by 4 .

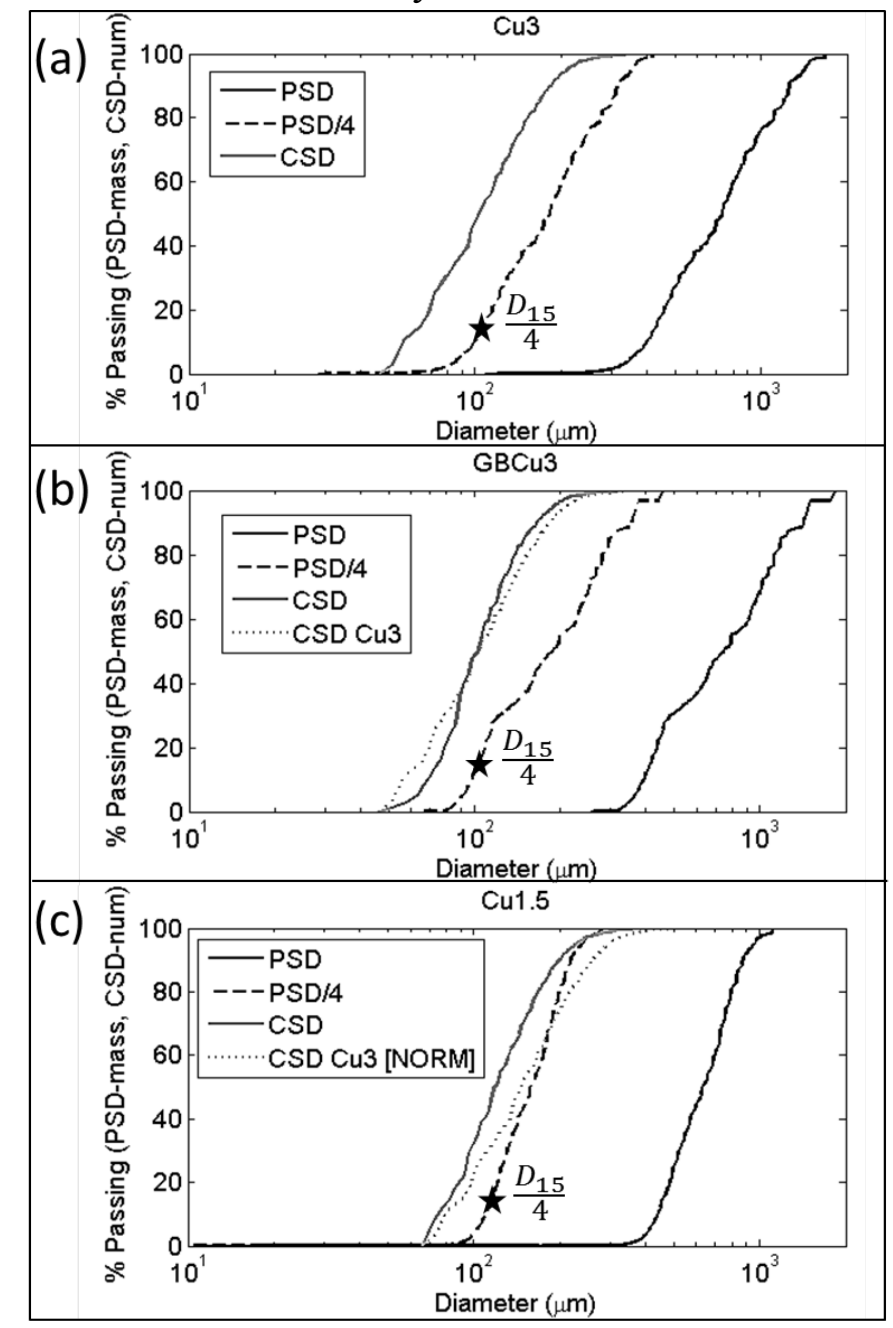

Figure 4: CSD relative to PSD, a) $\mathrm{Cu} 3$, b) $\mathrm{GBCu} 3$, c) $\mathrm{Cu} 1.5$

In terms of the Kézdi criterion, $D_{15} / 4$ corresponds to approximately the $50^{\text {th }}$ percentile of the constriction sizes for all three materials. This result is in good agreement with the result found by Shire et al. (2012), which was based on idealised spherical particles using DEM, and confirms that $D_{15} / 4$ represents 
a conservative estimate of the 'smaller constrictions' produced by a PSD.

An alternative way to interpret the results is to assume that $D_{15} / 4$ is intended to represent the $15^{\text {th }}$ percentile of the CSD $\left(D_{c 15}\right)$, but includes a factor of safety. The measured ratios between $D_{15}$ and $D c 15$ ranged from approximately 5.5 to 6.5 . These values are in good agreement with experimental results in the literature (Bertram 1940; Fannin \& Moffat 2006) which suggested stability up to $D_{15} / d_{85}$ values of 5 to 6 . This supports the physical interpretation that the factor 4 in the Kézdi criterion represents simply the ratio of particle to constriction sizes, with a factor of safety of roughly 1.5 .

The Kenney \& Lau criterion is applied over a wider portion of the PSD and hence the full shape of the CSDs relative to the PSDs should be considered. The CSD for the $\mathrm{Cu} 3$ material (Figure 4(a)) is roughly parallel to the PSD, with a size ratio of approximately 1:6.5 relating a point on the PSD to the same percentile in the CSD. Comparing the solid and dotted grey lines in Figure 4(b), the GBCu3 material (spherical glass beads) produces a more uniform distribution of constriction sizes than the $\mathrm{Cu} 3$ material (sub-angular sand, shown as a dashed line). For $\mathrm{GBCu} 3$ the size ratio between constrictions and particles ranges from around 1:5 to 1:6.5. In Figure 4(c), the dotted grey line shows the CSD for $\mathrm{Cu} 3$, normalised to have the same minimum constriction size as $\mathrm{Cu} 1.5$. While $\mathrm{Cu} 1.5$ clearly produces a narrower range of constriction sizes than $\mathrm{Cu} 3$, the CSD is not parallel to the PSD and the largest constriction sizes have a ratio of almost 1:4 to the particle sizes. Again, if the factor of 4 in the Kenney \& Lau criterion is interpreted as the ratio of particle to constriction sizes, with a factor of safety, then that factor of safety is typically around 1.3 to 1.6 , but may be as low as 1.0 for the largest constrictions in uniform materials.

Shire (2014) showed using DEM that when $C_{u}$ is increased above 3 the CSD, normalized by $D_{0}$ of the particles, remains the same. Hence for higher $C_{\mathrm{u}}$ values the factor between particle and constriction sizes will remain constant at around 6.5 for the smallest constrictions and will gradually increase above 6.5 for the largest constrictions.

The factor between particle and constriction sizes should not be thought of as giving a unique constriction size. Instead multiplying a particle size by this factor indicates that, somewhere in the material there will be constrictions of this size, but there will also be many larger than this size. In the next section laboratory tests are used to show how far fine particles can travel before being retained by a suitably small constriction.

\section{LABORATORY FILTER TESTS}

\subsection{Test procedure}

Soria et al. (1993) presented the methodology for a simple filter test, as an experimental means to estimate CSD. The thickness of a filter is incrementally increased; the filter thickness is used to estimate the number of constrictions passed, based on simple analytical assumptions; then the size distribution of particles which pass through the filter is used to estimate the constriction size distribution. Wu et al. (2012) replicated the same test procedure but, by comparing the results with DEM simulations, found that determining the CSD is challenging as it is very sensitive to the assumed spacing between constrictions. In the current study a similar test procedure was followed but, rather than trying to determine CSD (which can be measured much more accurately from microCT images), the tests were used to observe the extent to which fine particles move before they are retained in filters with $\mathrm{D}_{15} / \mathrm{d}_{85}<<4$, i.e. where the filter should easily be able to retain the fine material.

Referring to Figure 5, some minor alterations were made from the apparatus described by Soria et al. (1993) and Wu et al. (2012), to resolve practical issues with the test operation. Whereas the original apparatus constructed the filter material to a given thickness within a cylinder, achieving an evenly compacted and level layer only a few millimeters thick is extremely challenging, so the modified apparatus includes a separate sample chamber of the correct thickness, which was screwed onto the base of the test cylinder and separated from it by a taut nylon mesh. The cylinder was inverted; the filter material was placed in the chamber, compacted and levelled; a standard sieve tray (the type commonly used to determine PSDs) was placed over the filter, restrained laterally by flexible rubber tubing and supported vertically by a stiff perforated plate; then the apparatus was re-inverted and placed over a bucket, resulting in the configuration shown in Figure 5. The cylinder was filled with water (supplied directly from a tap, taking care not to erode the surface of the filter) up to a head of $8 \mathrm{~cm}$ above the filter; the base suspension (6 $\mathrm{g}$ of fine material suspended in a beaker of water) was poured evenly over the water surface; then the water supply was stopped and the water in the cylinder allowed to percolate through the filter. Further details of the test procedure can be found in Nogues-Herrero (2015). 


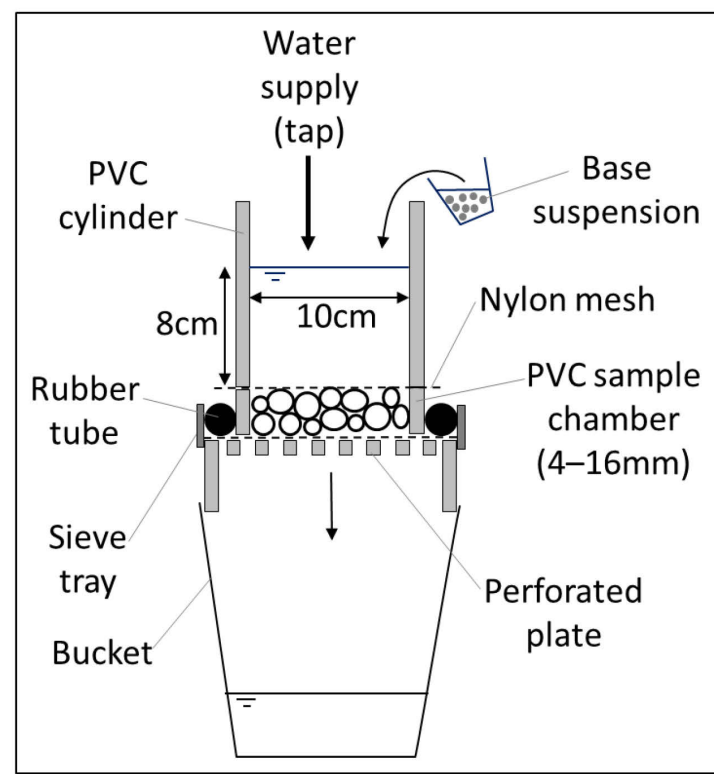

Figure 5: Schematic of modified laboratory filter test

Any fine material which passed through the filter into the bucket was dried and weighed. The proportion of material $<106 \mu \mathrm{m}$ in diameter was determined by dry sieving, while the PSD for the material $>106 \mu \mathrm{m}$ was determined using a QicPic laser scanner, as described in Altuhafi et al. (2013).

\subsection{Results: Effect of filter thickness}

Filter tests were performed using the materials $\mathrm{Cu} 3$, $\mathrm{GBCu} 3$ and $\mathrm{Cu} 1.5$ as the filter, with filter thicknesses of $4 \mathrm{~mm}, 9 \mathrm{~mm}$ and $16 \mathrm{~mm}$. To equate these thicknesses to the number of constrictions passed, the number of constrictions per unit volume was counted from the microCT images. Assuming the void space to be approximately isotropic and homogeneous, the cube root of the number per unit volume gives a rough approximation of the number per unit length, which was used to estimate the numbers of constrictions passed for each filter, as shown in Table 1.

Table 1. Filter thicknesses.

\begin{tabular}{|c|c|c|c|c|}
\hline & & \multicolumn{4}{|c|}{ Filter thickness } \\
\cline { 3 - 5 } Filter & \multirow{2}{*}{$\begin{array}{c}\text { No. of constrictions } \\
\text { material }\end{array}$} & per $\mathrm{mm}^{3}$ (microCT) & $4 \mathrm{~mm}$ & \multicolumn{3}{|c|}{$9 \mathrm{~mm}$} & $16 \mathrm{~mm}$ \\
\cline { 3 - 5 } & & \multicolumn{3}{|c|}{$\begin{array}{c}\text { Approximate no. of } \\
\text { constrictions passed }( \pm 5)\end{array}$} \\
\hline $\mathrm{Cu} 3$ & 25 & 10 & 25 & 45 \\
\hline $\mathrm{GBCu} 3$ & 27 & 10 & 25 & 50 \\
\hline $\mathrm{Cu} 1.5$ & 15 & 10 & 20 & 40 \\
\hline
\end{tabular}

In accordance with the procedure used by Soria et al. (1993) and $\mathrm{Wu}$ et al. (2012), the base material was selected to have $d_{100}$ equal to the $D_{0}$ of the filter, producing $D_{15} / d_{85}$ values as low as 1.5 to 2 . The smallest base particle were approximately $D_{0} / 6.5$. It should be noted that the test procedure was developed to capture the 'first pass' of fine particles through the filter, i.e. there is insufficient fine material to experience self-filtering (Soria et al. 1993).

The results for the mass of fines passing each filter are given in Table 2, and the PSDs of the passing material are shown in Figure 6. Note that the sudden changes in post-filter PSDs at $106 \mu \mathrm{m}$ occur because the total percentage passing the $106 \mu \mathrm{m}$ sieve has been combined with the laser scanning data for the particles $>106 \mu \mathrm{m}$.

Table 2. Percentage of base passing through filters.

\begin{tabular}{|c|c|c|c|c|}
\hline \multirow{2}{*}{$\begin{array}{c}\text { Filter } \\
\text { material }\end{array}$} & \multirow{2}{*}{$\mathrm{D}_{15} / \mathrm{d}_{85}$} & \multicolumn{3}{|c|}{ Filter thickness } \\
\cline { 3 - 5 } & & $4 \mathrm{~mm}$ & $9 \mathrm{~mm}$ & $16 \mathrm{~mm}$ \\
\cline { 3 - 5 } & 2 & 47 & 29 & 9 \\
\hline $\mathrm{Cu} 3$ & 2 & 36 & 27 & 16 \\
\hline $\mathrm{GBCu} 3$ & 1.5 & 11 & 4 & 3 \\
\hline $\mathrm{Cu} 1.5$ & 1.5 & \multicolumn{3}{|c}{} \\
\hline
\end{tabular}

For the $\mathrm{Cu} 34 \mathrm{~mm}$ and $9 \mathrm{~mm}$ filters there was significant movement of fine material (29-47\%). Figure 6(a) shows that the range of constriction sizes (dotted line) is roughly the same as the range of base particle sizes (solid black line) and the PSDs for material passing the filters (grey lines) indicate there was movement of particles across the whole size range, passing through 10-25 constrictions, despite the filter-base combination having a $D_{15} / d_{85}$ value of only 2 (well within the limiting value of 4 ). The $16 \mathrm{~mm}$ filter was sufficient to retain over $90 \%$ of the base material, proving that the filter-base combination would definitely be stable in a practical application, where granular filters may be several meters thick.

For the Cu1.5 filter, which has a $D_{15} / d_{85}$ value of only 1.5 , there are some base particles larger than the maximum constriction size in the CSD (Figure 6(c)) and both the mass and post-filter PSDs indicate that the movement of fine particles has been almost completely arrested, even for the $4 \mathrm{~mm}$ filter. 


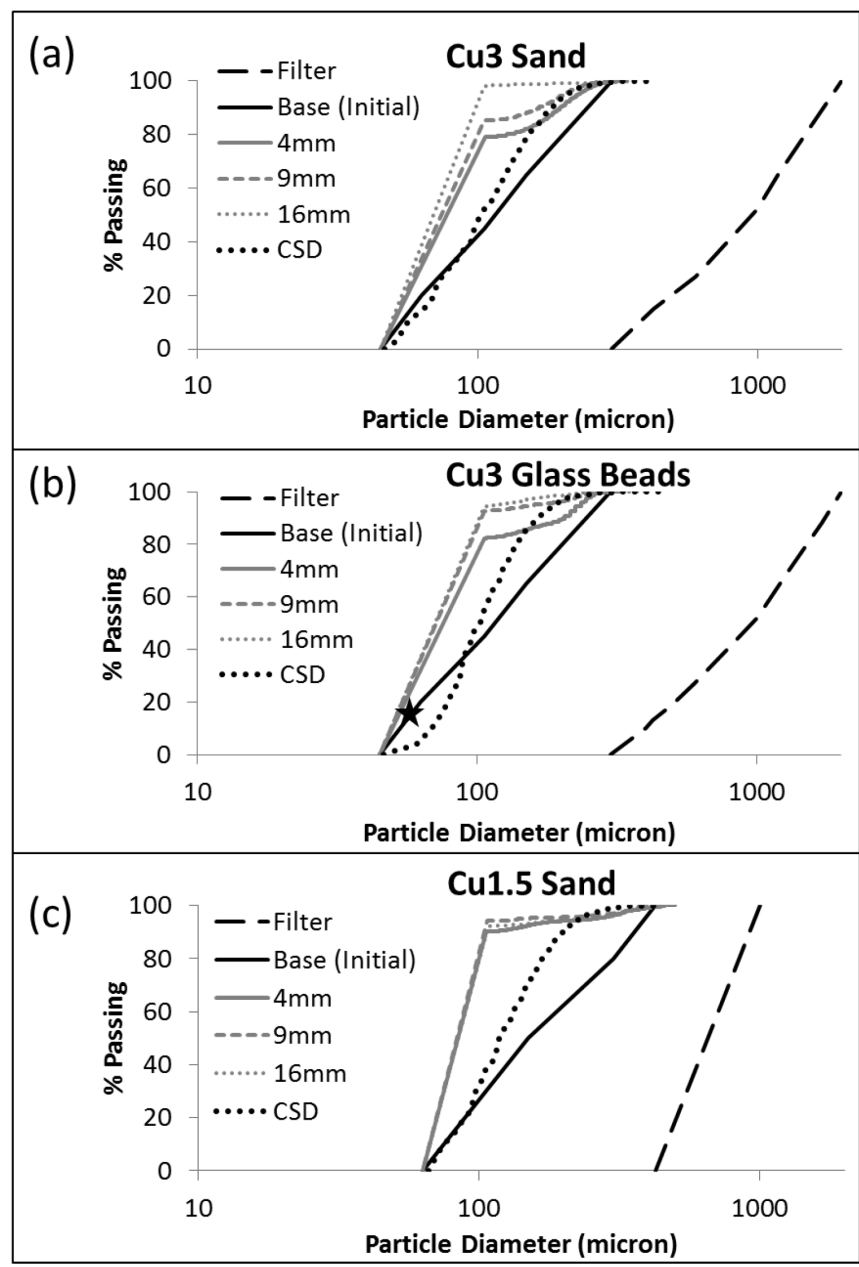

Figure 6: PSDs for filter, base and material passing through filter, a) $\mathrm{Cu} 3$ filter, b) $\mathrm{GBCu} 3$ filter, c) $\mathrm{Cu} 1.5$ filter.

Referring to Figure 6(b) and Table 2, at first glance the $4 \mathrm{~mm}$ and $9 \mathrm{~mm}$ results for the $\mathrm{GBCu} 3$ suggest that it is a more effective filter than $\mathrm{Cu} 3$, in terms of both the mass passing and the post-filter PSDs, even though the $D_{15} / d_{85}$ value is the same for $\mathrm{GBCu} 3$ and $\mathrm{Cu} 3$. This improvement corresponds to a difference at the upper end of the CSDs (GBCu3 has less large constrictions than $\mathrm{Cu} 3$ in Figure 4(b)) and also a small increase in the frequency of constrictions in $\mathrm{GBCu} 3$ relative to $\mathrm{Cu} 3$ (Table 1). However closer inspection of the $16 \mathrm{~mm}$ results shows that $16 \%$ of the base material is still passing the filter. Referring to Figure 6(b), the CSD for the GBCu3 material has a 'tail' at the lower end, indicating a lack of very small constrictions. The black star in Figure 6(b) indicates the smallest $16 \%$ of the base material by mass and this corresponds with the position of the tail in the CSD, suggesting that this very fine material may be moving freely through the filter, as it is smaller than the smallest constriction size. Given that this material has passed $16 \mathrm{~mm}$ (roughly 50 constrictions) through the filter, it is likely that it will continue to move freely through any length of filter.

These results for GBCu3 have two important implications. Firstly they show that the distance travelled by fine particles before they are retained can be very different for two cases with the same $D_{15} / d_{85}$ value (e.g. GBCu3 and Cu3). Secondly they show that without self-filtering by the fine particles, filter instability or suffusion could occur at $D_{15} / d_{85}$ values as low as 2 and hence self-filtration is absolutely necessary for the Kézdi (1979) criterion to give safe results. The Kenney \& Lau (1985) method automatically checks for self-filtering of fine particles so this is not an issue.

\section{CONCLUSIONS}

Two of the most commonly used criteria for suffusion, Kézdi (1979) and Kenney \& Lau (1985) include an empirically derived factor of 4 between the size of fine and coarse particles, which is commonly justified using simple filter rules. This paper used 3D microCT images of sands and glass beads, as well as laboratory filter tests on the same materials, to interpret the physical significance of this factor 4 in the underlying filter rules.

For the Kézdi (1979) criterion:

- Constriction sizes distributions (CSDs - Figure 4) indicate a typical factor of 5.5 to 6.5 between the size of coarse particles and void constrictions. Values may be as low as 4 for the largest constrictions in uniform materials.

- These results suggest that for $D_{15} / d_{85}<4$ in the Kézdi (1979) criterion, the number 4 is used to relate $D_{15}$ to the $15^{\text {th }}$ percentile of the constriction sizes, applying a factor of safety of roughly 1.5.

- The results agree closely with previous experimental studies (Bertram 1940; Fannin \& Moffat 2006) which showed that suffusion does not occur until $D_{15} / d_{85}$ values greater than 5 or 6 are reached.

- Laboratory filter tests showed that in cases with $D_{15} / d_{85}$ as low as 2 , fine particles could move through 10-25 constrictions before being retained and $D_{15} / d_{85}$ had to be lowered to 1.5 before this movement was completely arrested.

- Because fine particles were not able to self-filter, it was possible to observe unrestrained movement of fine particles at $D_{15} / d_{85}=2$, even though this should be stable according to the $D_{15} / d_{85}<4$ criterion. Hence the Kézdi (1979) criterion, despite being conservative in terms of constriction sizes, may be unsafe if the fines are not able to self-filter.

For the Kenney \& Lau (1985) criterion:

- The method chooses to assess $F$ and $H$ values at sizes $D$ and $4 D$ (Figure 1(b)) and here the number 4 appears to signify the size ratio between coarse particles and void constrictions, and includes a factor of safety of 1.0 to 1.6 , but typically around 1.5.

- This method automatically checks for selffiltration of the fine material and hence the issues identified by laboratory filter tests are not a concern for this criterion. 


\section{ACKNOWLEDGEMENTS}

The authors would like to thanks Alan Bolsher for his assistance in modifying the laboratory filter test. This work was undertaken as part of $\mathrm{PhD}$ research funded by the EPSRC under a DTG scholarship.

\section{REFERENCES}

Altuhafi, F., O’Sullivan, C. \& Cavarretta, I., 2013. Analysis of an image based method to quantify the size and shape of sand particles. Jour. Geotechnical and Geoenvironmental Engineering, 139(8), pp.1290-1307.

Bertram, G.E., 1940. An experimental investigation of protective filters. In Soil Mechanics Series No.7. Cambridge MA: Graduate School of Engineering, Harvard University.

Cnudde, V. \& Boone, M.N., 2013. High-resolution X-ray computed tomography in geosciences: A review of the current technology and applications. Earth-Science Reviews, 123, pp.1-17..

Fannin, R.J., 2008. Karl Terzaghi: From theory to practice in geotechnical filter design. Journal of Geotechnical and Geoenvironmental Engineering, ASCE, 134(3), pp.267276.

Fannin, R.J. \& Moffat, R.A., 2006. Observations on internal stability of cohesionless soils. Geotechnique, 56(7).

Fonseca, J. et al., 2012. Non-invasive characterisation of particle morphology of natural sands. Soils and Foundations, 52(4), pp.712-722.

Foster, M., Fell, R. \& Spannagle, M., 2000. The statistics of embankment dam failures and accidents. Canadian Geotechnical Journal, 37,5, pp.1000-1024.

Ishihara, K., 1993. Liquefaction and flow failure during earthquakes. Géotechnique, 43(3), pp.351-451.

Kenney, T.C. \& Lau, D., 1985. Internal stability of granular filters. Canadian Geotechnical Journal, 22, pp.215-225.

Kézdi, A., 1979. Soil Physics: Selected Topics,

Nogues-Herrero, R., 2015. Internal erosion tests to relate particle size distribution to the stability of selected granular filters. Imperial College London.

Otsu, N., 1979. A Threshold Selection Method from GrayLevel Histograms. IEEE Transactions on Systems, Man, and Cybernetics, 9(1), pp.62-66.

Ronnqvist, H., 2015. Predicting suffusion potential of dam core soil of glacial till. In Proc. of TKZ2015, Wintera J. \& Wity A. (eds.), Eksploatacja budowli piętrzacych - diagnostyka $i$ zapobieganie zagrożeniom, . pp. 115-126.

Shire, T., 2014. Micro-scale Modelling of Granular Filters. PhD Thesis, Imperial College London.

Shire, T., Hanley, K.J. \& Fannin, R.J., 2014. Fabric and Effective Stress Distribution in Internally Unstable Soils. Journal of Geotechnical and Geoenvironmental Engineering, pp.1-11.

Shire, T., Sullivan, C.O. \& Gaudray, G., 2012. Comparison of two methods for measurement of soil constriction size distribution. In International Conference on Scour and Erosion, Paris. pp. 249-256.

Skempton, A.W. \& Brogan, J.M., 1994. Experiments on piping in sandy gravels. Geotechnique, 44, No. 3, pp.449-460.

Soria, M.H.., Aramaki, R.T. \& Viviani, E., 1993. Experimental determination of void size curves. In Filters in Geotechnical and Hydraulic Engineering, Balkema, Rotterdam. pp. 43-48.
Taylor, H.F., O’Sullivan, C. \& Sim, W.W., 2015. A new method to identify void constrictions in micro-CT images of sand. Computers and Geotechnics, 69, pp.279-290.

Vincens, E., Witt, K.J. \& Homberg, U., 2014. Approaches to Determine the Constriction Size Distribution for Understanding Filtration Phenomena in Granular Materials. Acta Geotechnica - PREPRINT.

$\mathrm{Wu}$, L. et al., 2012. Laboratory experiments for the determination of the constriction size distribution of granular filters. International Conference on Scour and Erosion, pp.233-240. 